Revista de Ciencias Sociales - Número 63 (2013) - Páginas 169-181

Presidio perpetuo irreductible como pena inhumana y degradante:análisis...

\title{
PRESIDIO PERPETUO IRREDUCTIBLE COMO PENA INHUMANA Y DEGRADANTE: ANÁLISIS DEL CASO VINTER Y OTROS V. REINO UNIDO (TRIBUNAL EUROPEO DE DERECHOS HUMANOS)
}

\author{
IRREDUCIBLE LIFE SENTENCE AS AN INHUMANE \\ AND DEGRADING PUNISHMENT: ANALYSIS OF \\ VINTER AND OTHERS V. UNITED KINGDOM \\ (EUROPEAN COURT OF HUMAN RIGHTS)
}

PABLO CONTRERAS V.*

Universidad Alberto Hurtado pcontreras@nlaw.northwestern.edu

\section{Resumen}

El trabajo analiza la sentencia Vinter y otros v. Reino Unido, del Tribunal Europeo de Derechos Humanos. El Tribunal estimó que la pena de presidio perpetuo sin posibilidad de beneficios o salidas penitenciarias, constituía una pena inhumana y degradante. El paper examina los grados de discreción que tienen los Estados en la configuración de sus sistemas de justicia criminal y

* Abogado. Licenciado en Ciencias Jurídicas y Magíster en Gobierno y Sociedad, Universidad Alberto Hurtado; Master en Derecho (LL.M.) y candidato a Doctor en Derecho (S.J.D.), Northwestern University. Artículo recibido el 30 de octubre de 2013 y aceptado el 11 de noviembre de 2013.

Revista de Ciencias Sociales - Número 63 (2013) - Universidad de Valparáíso - ISSN 0716-7725-Valparaíso, Chile 
cuáles son los estándares que el Tribunal Europeo fija a efectos de cumplir con las obligaciones del artículo 3 de la Convención Europea de Derechos Humanos. El texto concluye que el Tribunal ha elevado los estándares de protección frente al ius puniendi estatal, en relación con el alcance de las penas de presidio.

\section{Palabras claves}

Presidio perpetuo, pena inhumana y degradante, proporcionalidad.

\section{Abstract}

The paper reviews the decision Vinter and others $v$. United Kingdom, of the European Court of Human Rights. The Court ruled that life imprisonment without parole constituted an inhumane and degrading punishment. The text examines what degrees of discretion do States have in the configuration of their own criminal justice systems. It also examines the standards fixed by the Court, concerning article 3 of the European Convention of Human Rights. The paper concludes that the Court has elevated the human rights standards before the State's ius puniendi when it imprisons someone.

\section{Keywords}

Life imprisonment, inhumane and degrading punishment, proportionality.

\section{Introducción}

En los últimos años, las penas de presidio o reclusión perpetua han sido sometidas al escrutinio de importantes Cortes nacionales e internacionales. En materia de reclusión perpetua, dos sentencias deben destacarse: primero, Miller v. Alabama ${ }^{1}$, de la Corte Suprema federal de Estados Unidos, prohibiéndose la imposición obligada del presidio perpetuo a menores, sin posibilidad de libertad condicional; segundo, el caso Mendoza y otros v. Argentina ${ }^{2}$, dictada por la Corte Interamericana

1. Corte Suprema federal de Estados Unidos, Miller v. Alabama, 132 SCt 2455 (2012).

2. Corte Interamericana de Derechos Humanos, Mendoza y otros v. Argentina, Sentencia de excepciones preliminares, fondo y reparaciones, Mayo 14, 2013, Ser. C, $\mathrm{N}^{\circ} 260$.

Facultad de Derecho y Ciencias Sociales - Universidad de Valparaíso - Chile 
de Derechos Humanos, declarándose que la imposición del presidio perpetuo a menores de edad constituye un castigo desproporcionado que viola la prohibición de tratos crueles, inhumanos o degradantes, del artículo 5.2 de la Convención Americana. Ambas decisiones versan sobre los derechos de los menores de edad frente al ejercicio del ius puniendi estatal. Sin embargo, sus alcances difieren considerablemente. La primera decisión - Miller - sólo llegó al punto de prohibir esquemas legales que obligan la imposición del presidio perpetuo cuando se cometen determinados crímenes, siempre y cuando no exista la posibilidad de libertad condicional (parole) ${ }^{3}$; por ello, no estableció una prohibición absoluta de dicha pena cuando el sentenciado es un menor de edad. Eso fue, precisamente, lo que falló la Corte Interamericana en Mendoza: la desproporcionalidad de las penas de presidio perpetua impuestas a menores de edad constituyeron tratos crueles e inhumanos, en violación a lo dispuesto en el artículo 5 de la Convención Americana de Derechos Humanos'.

Sin embargo, un salto cualitativamente relevante dio recientemente la Gran Sala del Tribunal Europeo de Derechos Humanos ("TEDH”), al fallar el caso Vinter y otros v. Reino Unido. En Vinter, debía decidirse si la imposición de la pena de presidio perpetuo a un individuo, sin posibilidad o posibilidad de recuperar la libertad, viola la prohibición de tortura y de penas o tratos inhumanos o degradantes, establecida en el artículo 3 del Convenio Europeo de Derechos Humanos (“CEDH” o "Convención”). El TEDH estimó que Reino Unido había violado la Convención y con ello elevó el estándar europeo para las penas privativas de libertad, como es el presidio perpetuo, y fijó un nuevo horizonte en el ámbito del derecho internacional de los derechos humanos.

3. BHARAT, Malkani: "Sentencing Children Who Kill: One Giant Leap for the US Supreme Court, One Small Step for International Human Rights Law”, en: Human Rights Law Review (Vol. 12, No 4), 2012. Págs. 801 y 805-806.

4. Mendoza v. Argentina, cit., 183.

5. TEDH, Vinter and others v. United Kingdom, App. N ${ }^{\text {os. }} 66069 / 09,130 / 10$ y 3896/10, Jul. 9, 2013.

Revista de Ciencias Sociales - Número 63 (2013) - Universidad de Valparáíso - ISSN 0716-7725-Valparaíso, Chile 
En este comentario analizaremos el problema y la cuestión jurídica sometida al TEDH, en relación con el caso concreto (II). A partir de ello, se revisa las consideraciones que adopta, por un lado, respecto de la discreción de los Estados en materia de justicia criminal y la pena en comento (III) y, por el otro, en relación al estándar y condiciones que fija para que el presidio perpetuo sea compatible con la prohibición de penas inhumanas de la CEDH (IV). La última parte del comentario presenta las conclusiones y conjetura sobre el nuevo estándar aplicable al artículo $3 \mathrm{CEDH}$ para otros regímenes de derechos humanos (V).

\section{El problema}

La decisión en Vinter agrupa las situaciones de tres individuos que, en Reino Unido, fueron condenados a una pena perpetua de privación de libertad. En determinadas circunstancias, el juez que decide la pena puede imponer una orden que priva de libertad al sujeto de por vida ("whole life order") y éste sólo puede ser excarcelado o liberado por decisión adoptada por el Secretario de Estado por razones compasivas ${ }^{6}$. Tal discreción había sido ejercida excepcionalmente en el pasado, en casos de enfermedades terminales o grave incapacidad ${ }^{7}$. Si bien la legislación nacional permite que el juez pueda definir un plazo mínimo de reclusión sin revisión previa, también lo autoriza a imponer una orden de privación de libertad de por vida. Dentro los criterios que exige la legislación para decretar tal orden, se especifica que es una medida reservada para delitos cuya gravedad es "excepcionalmente alta" y se detallan, por vía ejemplar, casos de homicidios que responden a tal calificación ${ }^{8}$.

La posibilidad de liberación anticipada depende de la discreción del Secretario de Estado. Para ello, conforme a la legislación inglesa, se

6. Id., 12 .

7. Marek Szydlo (2012): “Vinter v. United Kingdom”, en The American Journal of International Law (Vol. 106, No 3), p. 624.

8. $\quad$ Vinter, cit., 38-9.

Facultad de Derecho y Ciencias Sociales - Universidad de Valparaíso - Chile 
deben acreditar "circunstancias excepcionales que justifiquen la liberación del prisionero por razones compasivas". Los lineamientos políticos para una decisión de esa envergadura han sido definidas por Reino Unido. Básicamente, el individuo debe estar/encontrarse en una situación extrema: sufriendo una enfermedad terminal en la que la muerte es una posibilidad cierta y cercana en el tiempo, pero donde además el riesgo de reincidencia es mínimo, el mantener la reclusión reduciría la expectativa de vida, existen adecuados cuidados sanitarios afuera de la prisión para el individuo y, finalmente, la liberación anticipada generaría un significativo beneficio para el prisionero o su familia ${ }^{10}$. Como se puede observar, se trata de una hipótesis sumamente excepcional y exigente, a efectos de autorizar la liberación de un reo penado con presidio perpetuo.

El TEDH debía decidir si la imposición de una orden que priva de libertad a un individuo de por vida era compatible con la prohibición de penas inhumanas o degradantes del artículo 3 de la Convención. Para ello, procede a revisar el estado de la legislación y jurisprudencia nacional y comparada. Particularmente relevante para su análisis serán las consideraciones que en su momento adoptó el Tribunal Constitucional Federal Alemán —en los casos Cadena Perpetua ${ }^{11}$ y Criminal de Guerra ${ }^{12}$ - y lo dispuesto en el Pacto Internacional de Derechos Civiles y Políticos y la interpretación que del mismo ha hecho el Comité de Derechos Humanos ${ }^{13}$.

$$
\begin{array}{r}
\text { 9. Id., } 42 . \\
\text { 10. Id., } 43 .
\end{array}
$$

11. BVerfGE 45, 187 (1977) [Life Imprisonment]. Una traducción del caso al español puede encontrarse en Jürgen Schwabe (2009): Jurisprudencia del Tribunal Constitucional Federal Alemán (México, Konrad Adenauer Stiftung). Págs. $54 \mathrm{y} \mathrm{ss.}$
12. BVerfGE 72, 105 (1986) [War Criminal].
13. Véase Comité de Derechos Humanos (1992): $§ 10$.

Revista de Ciencias Sociales - Número 63 (2013) - Universidad de Valparáíso - ISSN 0716-7725-Valparáiso, Chile 


\section{Discreción de los Estados Partes en materia de justicia criminal}

Los Estados partes cuentan con una discreción en la regulación y protección interna de los derechos establecidos en la CEDH. Se trata de un corolario lógico de la estructura internacional de protección regional e internacional de los derechos humanos. La maquinaria de protección internacional sólo entra en juego una vez que se han agotado los recursos y acciones domésticos. En esta materia, la discreción apunta, por un lado, a la conformación soberana de sistemas de justicia criminal y, por el otro, al establecimiento de garantías de derechos frente al ius puniendi estatal. La discreción, sin embargo, no es absoluta y se encuentra sometida al control y revisión de los órganos internacionales de derechos humanos, como es el TEDH.

Para fallar el asunto sometido a su conocimiento, el TEDH resuelve articular ciertos principios básicos que guían la intensidad del escrutinio judicial. Ello, como contrapartida, fija la esfera de discreción que tienen los Estados Partes bajo las obligaciones que impone el CEDH. Específicamente en este caso, el TEDH delinea dos elementos centrales que circunscriben su propia competencia. El primer elemento consiste en que los Estados tienen una esfera de discreción para configurar los sistemas penales nacionales. El segundo elemento dice relación con la elección de la sanción penal y su duración, cuestiones que son de determinación discrecional de los Estados. Revisaremos ambos elementos a continuación.

En primer lugar, la sentencia declara que cada Estado puede decidir sobre las particularidades de su propio sistema de justicia criminal, "incluyendo la revisión de sentencias y los arreglos de liberación”; estas materias, en principio, se encuentran fuera del margen de supervisión del TEDH, salvo que la regulación doméstica contravenga algunos de los principios de la Convención ${ }^{14}$. Este principio había sido establecido previamente en Kafkaris v. Chipre ${ }^{15}$. La configuración específica del sistema penal es, por tanto, materia que

14. Vinter, cit., 104 .

15. App. $\mathrm{N}^{\text {o }}$ 21906/04, Feb. 12, 2008, 99.

Facultad de Derecho y Ciencias Sociales - Universidad de Valparaíso - Chile 
discrecionalmente fijan los Estados. En principio, el TEDH no tiene jurisdicción para evaluar las reglas que articulen dicho sistema, en particular, aquellas que definan la revisión de condenas y los beneficios penitenciarios para reos. Cada Estado sería soberano, entonces, para responder a las necesidades punitivas a través de los medios que les sean necesarios. No obstante, tal discreción —en sus concreciones específicas- puede ser supervisada si existe una presunta vulneración de las obligaciones del CEDH.

En segundo lugar, el TEDH confirma que la decisión de la duración apropiada de una pena privativa de libertad es una materia que le corresponde a las autoridades nacionales de los Estados Partes. En este punto, la referencia al margen de apreciación es explícita ${ }^{16}$. La declaración del TEDH no hace sino confirmar la subsidiariedad estructural de los sistemas regionales de derechos humanos ${ }^{17}$. En muchos casos, la subsidiariedad "resultará en un grado de discreción sobre la interpretación e implementación de los derechos [...]"18. Aplicado a Vinter, la decisión sobre la pena y su duración es algo que queda reservado, en principio, a la discreción de los Estados. La revisión internacional opera en subsidio de la apreciación y protección que efectúan los órganos nacionales. En este nivel, las penas de presidio perpetuo no son incompatibles, per se, con la $\mathrm{CEDH}^{19}$.

La declaración, en este punto, se asemeja al estándar genérico de escrutinio fijado por el TEDH para supervisar el margen de apreciación de los Estados Partes de la Convención ${ }^{20}$. El mismo TEDH ha establecido que "la maquinaria de la protección [de la CEDH] es subsidiaria a los sistemas nacionales que protegen los derechos" ${ }^{21}$. Por

16. Vinter, cit., 105.

17. CAROZZA, Paolo G. Carozza: "Subsidiarity as a Structural Principle of International Human Rights Law”, en The American Journal of International Law (Vol. 97), 2003.

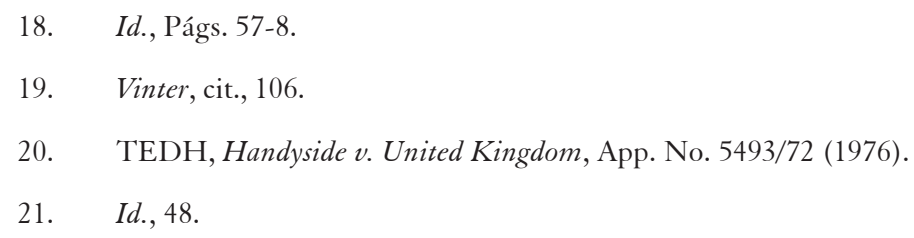


ello, la tarea de protección corresponde, en primer término, a los Estados Partes de la CEDH y, complementariamente, al TEDH ${ }^{22}$. La discreción está construida desde el punto de vista de la subsidiariedad, bajo la cual se estructuran las instancias regionales de protección de los derechos humanos ${ }^{23}$. En efecto, el juez nacional se encuentra conectado directamente con los hechos materia del juicio y los tribunales internacionales no son instancias de revisión del juicio de culpabilidad que hagan las cortes nacionales. Es relevante destacar que el TEDH, aún empleando doctrinas de autorrestricción judicial, mantiene su poder revisión final. Tal como ha señalado en numerosas oportunidades, la discreción nacional va acompañada de supervisión internacional ${ }^{24}$. La revisión internacional se aboca a la determinación de si ha existido una violación de las obligaciones internacionales del tratado en cuestión - como el CEDH o la Convención Americana- pero no de constituir una "cuarta instancia" de juzgamiento o de revisión penal ${ }^{25}$.

Sin embargo, la discreción estatal tiene límites en esta materia. El primero dice relación con una sentencia que impone una pena manifiestamente desproporcionada (grossly disproportionate) como sanción de una conducta determinada. En ese caso sí se verificaría una violación del artículo 3 de la Convención ${ }^{26}$. Se trata de una de las principales novedades de este fallo ${ }^{27}$. Pese a ello, el mismo TEDH admite que es difícil que acontezca una violación tal en un caso concreto. Por

22. $I d$.

23. CAROZZA, Paolo: cit. Pág. 62-3.

24. Handyside, cit., 49.

25. Sobre la doctrina de la prohibición de la "cuarta instancia”, véase RODRÍGUEZ PINZÓN, Diego: “The 'Victim' Requirement, the Fourth Instance Formula, and the Notion of 'Person' in the Individual Complaint Procedure of the Inter-American Human Rights System”, en: ILSA Journal of International and Comparative Law (Vol. 7), 2001.

26. Vinter, cit., 83, 88 y 89 .

27. SZYDLO, cit. Pág. 627.

Facultad de Derecho y Ciencias Sociales - Universidad de Valparaíso - Chile 
otro lado, la decisión en Vinter aporta un nuevo límite a esta discreción nacional: el estándar aplicable a las penas de presidio perpetuo, que se revisan en el siguiente apartado.

\section{El estándar aplicable: reductibilidad del presidio perpetuo}

La gran innovación en relación con el contenido protegido por el artículo 3 de la CEDH, es la prohibición de una pena de presidio perpetuo irreductible. Si bien, como anotamos anteriormente, el presidio perpetuo no viola, per se, la Convención, el TEDH ha estimado que tal incumplimiento sí se configura en el evento que el Estado no permita una posibilidad de liberación o salida ${ }^{28}$. La salida o liberación puede darse por diversos motivos, tales como la conmutación de la pena, su término anticipado o la libertad condicional, entre otros posibles beneficios penitenciarios. El TEDH no exige que sea una posibilidad cierta, pero sí exige que dicha posibilidad exista. La fundamentación radica en que la privación de libertad requiere que sirva a sus bases penológicas para permitir la reclusión del individuo ${ }^{29}$. Dichas bases pueden ser diversas: consideraciones retributivas de castigo, disuasión, protección pública o rehabilitación. Lo importante es que los órganos estatales efectúen un "balance entre estas justificaciones" y que su resultado "no es necesariamente estático [en el tiempo] y puede cambiar en el curso [de la ejecución] de la sentencia" 30 .

En esta línea, es relevante destacar que el TEDH asume la tesis del TCF alemán, que ha fallado como incompatible con la dignidad humana el privar de libertad a un individuo y no dar siquiera posibilidad alguna de revisión para que éste recupere su libertad. La práctica estatal es un elemento relevante al momento de determinar el estándar de escrutinio internacional, como algunos autores lo han demostrado ${ }^{31}$.

\footnotetext{
28. Vinter, cit., 109.

29. Vinter, cit., 111.

30. Id.

31. Según Legg, la práctica estatal es un factor externo que afecta el estándar de supervisión internacional. En términos generales, la ausencia de consenso

Revista de Ciencias Sociales - Número 63 (2013) - Universidad de Valparáíso - ISSN 0716-7725-Valparáiso, Chile
} 
En este caso, la mayoría de los Estados europeos no contemplaban penas de presidio perpetuo "irreductibles" —incluso para los delitos más graves-y, es más, algunos como Portugal, España o Noruega no establecen siquiera la pena de presidio perpetuo ${ }^{32}$. El contexto regional, si bien no lo explicita claramente, puede haber influido en una intensificación del estándar de escrutinio del TEDH.

El estándar de reductibilidad del presidio perpetuo fijado por el TEDH, se conecta con una fundamentación más amplia de las reglas de derechos humanos aplicables a las penas. Dentro de los fines de la pena, el Tribunal dispone que éstas no pueden servir únicamente fines de castigo, sino también deben apuntar a la rehabilitación de los individuos ${ }^{33}$. Se trata de una cuestión que se encuentra prescrito positivamente en otros tratados internacionales de derechos humanos - como el Pacto de Derechos Civiles y Políticos ${ }^{34}$ y la Convención Americana de Derechos Humanos ${ }^{35}$ - y que ha sido interpretado así por organismos internacionales, como el Comité de Derechos Humanos $^{36}$. Lo que hace el TEDH es integrar esta finalidad de la pena al conjunto de obligaciones de derechos humanos que fija la Convención. Esto es particularmente relevante en relación con la pena

regional en una materia incrementa la deferencia a los Estados Partes, la práctica estatal a favor del individuo denunciante aumenta el escrutinio del TEDH y la práctica estatal a favor del Estado Parte incrementa deferencia. Véase LEGG, Andrew: The Margin of Appreciation in International Human Rights Law (Oxford, Oxford University Press), 2012. Págs. 116 y ss.

32. SZYDLO, cit. Pág. 626.

33. Vinter, cit., 115 .

34. Art. 10(3): “[...] El régimen penitenciario consistirá en un tratamiento cuya finalidad esencial será la reforma y la readaptación social de los penados [...]”.

35. Art. 5(6): “[...] Las penas privativas de la libertad tendrán como finalidad esencial la reforma y la readaptación social de los condenados”.

36. Comité de Derechos Humanos (1992): §10 ("Ningún sistema penitenciario debe estar orientado a solamente el castigo; esencialmente, debe tratar de lograr la reforma y la readaptación social del preso").

Facultad de Derecho y Ciencias Sociales - Universidad de Valparaíso - Chile 
de presidio perpetuo, puesto que, ejecutado incondicionalmente y de manera irreductible, imposibilita la viabilidad de una rehabilitación del individuo. Así, los Estados que no establecen ningún tipo de posibilidad de revisión de las bases penológicas del presidio perpetuo no satisfacen el estándar obligado por el artículo 3 de la Convención ${ }^{37}$. En consecuencia, una pena de presidio perpetuo irreductible es considerada una pena cruel e inhumana respecto de las obligaciones del CEDH.

Finalmente, cabe señalar que el TEDH determina que la implementación nacional del estándar permite una serie de alternativas domésticas. Por ello, el Tribunal afirma que no es su tarea prescribir la forma en que debe conducirse una revisión — ya sea administrativa o judicial_ de las bases penológicas de la reclusión perpetua. Igualmente, "no le compete al Tribunal determinar cuándo tal revisión debe efectuarse" 38 . Lo que hace el TEDH es dejar a los Estados la determinación particular de las reglas sustantivas y procedimentales de la revisión para determinar la posibilidad de una salida o liberación. En definitiva, la obligación internacional conlleva una esfera de discreción importante, que siempre puede ser sujetada a la revisión del TEDH. Dicha conclusión fluye como implicancia de discrecionalidad nacional, a la luz de los principios generales que el Tribunal desarrolló al comienzo de su razonamiento en este caso.

\section{Conclusiones}

El TEDH ha dado un gran paso en materia de las obligaciones internacionales de derechos humanos aplicables a las penas, al prohibir la imposición incondicionada e irreductible del presidio perpetuo, como una pena cruel e inhumana. La tendencia internacional había ido restringiendo el presidio perpetuo respecto de menores de edad, como los casos Miller y Mendoza lo demuestran. El TEDH da un salto cualitativo al prohibir el presidio perpetuo irreductible, aún cuando se

\footnotetext{
37. Vinter, cit., 121.

38. Vinter, cit., 121.
}

Revista de Ciencias Sociales - Número 63 (2013) - Universidad de Valparáíso - ISSN 0716-7725-Valparaíso, Chile 
trate de personas mayores de edad. Pero, además, su argumentación va sentando bases importantes para limitar el ius puniendi estatal. Por un lado, sostiene que para mantener una pena privativa de libertad debe existir alguna base penológica. Por el otro, el razonamiento del Tribunal hace que se incluya un mandato de rehabilitación y readaptación social de los individuos sujetos a penas privativas de libertad. Con ello, complementa el catálogo obligacional del CEDH con una regla que ya había sido incorporada en otros tratados internacionales de derechos humanos.

Por otro lado, en su decisión, ha determinado esferas de discreción estatal que se explican por el carácter subsidiario de la protección regional de derechos humanos. Esta articulación es necesaria para mantener la lógica estructural del sistema europeo. Sin perjuicio de ello, ha avanzado en prohibir el presidio perpetuo irreductible y en asegurar la supervisión de penas que sean manifiestamente desproporcionadas (grossly disproportionate).

Es altamente probable que esta sentencia fije un nuevo horizonte para repensar críticamente las obligaciones de derechos humanos en relación con los sistemas criminales. En particular, la Corte Interamericana de Derechos Humanos suele ser recepticia a las innovaciones que fija el TEDH. Ya en Mendoza, la Corte se plegó a ciertas decisiones del TEDH para prohibir el presidio perpetuo aplicado a menores de edad. Con este nuevo estándar y con la base positiva de una exigencia de readaptación social dispuesta por la Convención Americana, la Corte podría configurar nuevas exigencias para los Estados americanos.

\section{Bibliografía}

CAROZZA, Paolo G.: "Subsidiarity as a Structural Principle of International Human Rights Law”, en: The American Journal of International Law (Vol. 97), 2003.

Comité de Derechos Humanos (1992): “Observación General No 21:

Trato Humano de las Personas Privadas de Libertad (Artículo 10)", Adoptado en su 44 a sesión y reimpreso en Recopilación de las Observaciones Generales y Recomendaciones Generales Adoptadas por

Facultad de Derecho y Ciencias Sociales - Universidad de Valparaíso - Chile 
Órganos Creados en virtud de Tratados de Derechos Humanos (HRI/GEN/ 1/Rev.9 (Vol. I) 27 de mayo de 2008).

LEGG, Andrew: The Margin of Appreciation in International Human Rights Law (Oxford, Oxford University Press), 2012. Págs. 116 y ss.

MALKANI, Bharat: Malkani: "Sentencing Children Who Kill: One Giant Leap for the US Supreme Court, One Small Step for International Human Rights Law", en Human Rights Law Review (Vol. 12, No 4). 2012. Págs. 801 y 805-806.

RODRÍGUEZ PINZÓN, Diego: “The 'Victim' Requirement, the Fourth Instance Formula, and the Notion of 'Person' in the Individual Complaint Procedure of the Inter-American Human Rights System", en: ILSA Journal of International and Comparative Law (Vol. 7), 2001.

SCHWABE, Jürgen: Jurisprudencia del Tribunal Constitucional Federal Alemán, Konrad Adenauer Stiftung, México. 2009.

SZYDLO, Marek: "Vinter v. United Kingdom", en: The American Journal of International Law (Vol. 106, No 3), 2012.

\section{Sentencias citadas}

Corte Suprema federal de Estados Unidos, Miller v. Alabama, $132 \mathrm{SCt}$ 2455 (2012).

Corte Interamericana de Derechos Humanos, Mendoza y otros v. Argentina, Sentencia de excepciones preliminares, fondo y reparaciones, Mayo 14, 2013, Ser. C, No 260.

Tribunal Europeo de Derechos Humanos, Vinter and others v. United Kingdom, App. No 66069/09, 130/10 y 3896/10, Jul. 9, 2013.

Tribunal Europeo de Derechos Humanos, Kafkaris v. Chipre, App. No 21906/04, Feb. 12, 2008. 